3 Adachi $W$, Mitsuishi $Y$, Terai $K$, et al. The association of HLA with young-onset keratoconus in Japan. Am J Ophthalmol 2002; 133:557-9.

4 Woodhouse NJ, Sakati NA. A syndrome of hypogonadisim, alopecia, diabetes mellitus, mental retardation, deafness and ECG abnormalities. J Med Genet 1983;20:216-19.

5 Gul D, Ozata M, Mergen H, et al. Woodhouse and Sakati syndrome (MIM 241080) report of a new patient. Clin Dysmorph 2000;9:123-5.

6 Morrison DA, Rosser EM, Claove C. Keratoconus associated with a chromosome 7, 11 translocation. Eye 2001;15:556-7.

\section{Retinal ischaemia in type 1 neurofibromatosis}

Systemic occlusive arteriopathy associated with type 1 neurofibromatosis (NF-1) has been well described in the aortic and cerebral vasculature $^{1}$; however, it is poorly documented in the retinal vasculature with, to our knowledge, only three reported patients whose retinal vasculopathy was diagnosed at a later sequelae stage. ${ }^{2-4}$ We report the case of a young woman with NF-1 who presented with acute macular ischaemia combined with diffuse retinal arterial occlusive disease.

\section{Case report}

A 26 year old woman with NF-1 presented with a sudden painless loss of vision in her left eye. Visual acuity was counting fingers left eye and 20/20 right eye. NF-1 had previously been diagnosed on the basis of multiple café au lait spots, plexiform neurofibromas, and a paternal history of NF-1. Left fundus examination revealed confluent cottonwool spots in the perifoveal area, focal dilatations and narrowings of retinal venules, and progressive enlargement of retinal arterioles towards the periphery. The right fundus showed only peripapillary myelinated nerve fibres (fig 1).

Fluorescein angiography (FA) of the left eye showed no delayed perfusion in the choriocapillaris or central retinal artery, but a delayed retinal arteriovenous filling time. It revealed occlusion of macular arterioles, retinal arteriovenous communications in the mid-periphery, and a relatively well perfused retinal periphery (fig 2 ).

Cerebral and cervical magnetic resonance imaging and colour Doppler ultrasonography showed no vascular flow abnormalities. Internal medical examination and laboratory results excluded other diseases associated with retinal microvasculopathies.

One month after the initial examination all the cottonwool spots disappeared, and sheathing appeared in a few veins. FA showed worsening of the retinal arteriolar occlusions in the mid-periphery (fig 2). A panretinal photocoagulation was subsequently performed. At 2 year follow up, left visual acuity remained unchanged, and the patient did not exhibit any preretinal fibrovascular proliferation.

\section{Comment}

To our knowledge, only three cases of retinal vascular occlusion in patients with NF-1 have been previously reported. All cases were diagnosed at a later sequelae stage. Moade et $a l^{2}$ and Kadoi et al, ${ }^{3}$ respectively, reported the cases of a child who presented with an unilateral amblyopia, and a young woman with a history of unilateral poor vision. They both presented with diffuse sheathing of retinal vessels, retinal arteriovenous
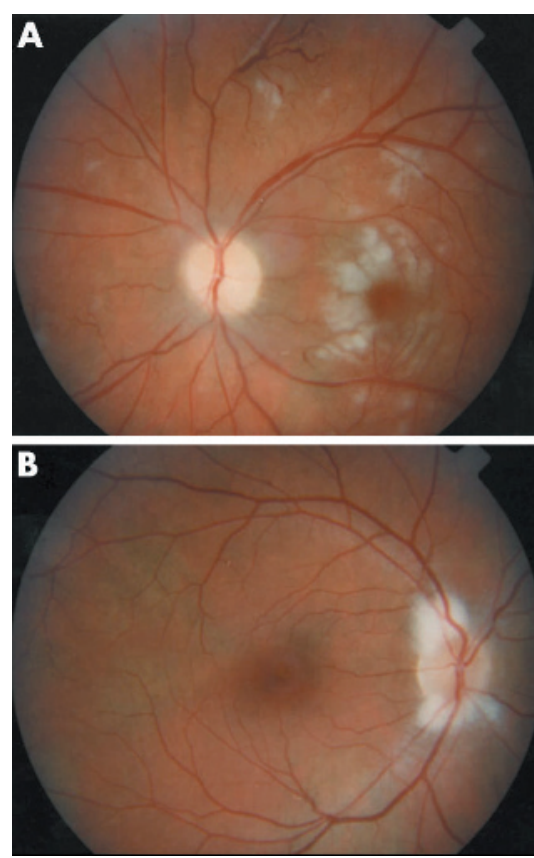

Figure 1 Fundus photographs of the left and the right eyes at presentation. (A) Colour fundus photograph of the left eye showing large confluent cottonwool spots in the perifoveal area. (B) Colour fundus photograph of the right eye showing peripapillary myelinated nerve fibres. (C) Red-free fundus photograph of the left eye. Upper periphery showing focal dilatations and narrowings of retinal veinules, and capillary rarefaction. Retinal arteriovenous communications are also present (arrows).
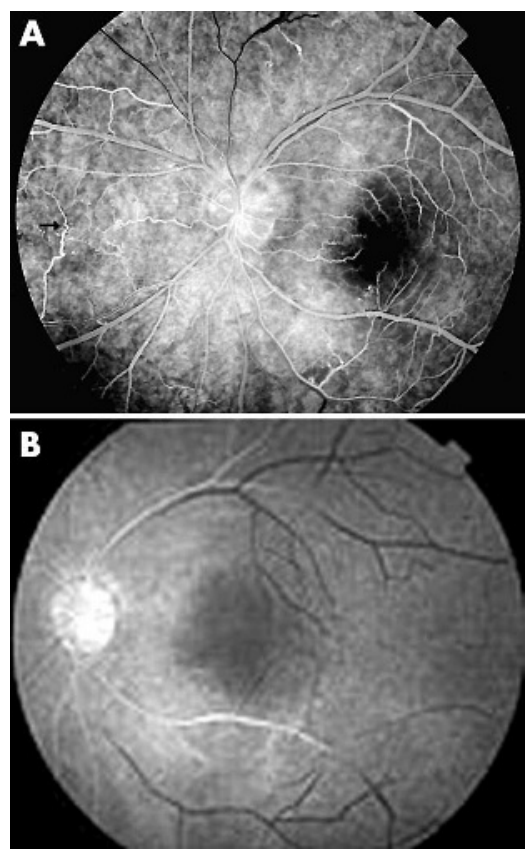

Figure 2 Fluorescein retinal angiography of the left eye, 40 seconds after dye injection, at presentation (A) and 1 month later (B). (A) Occlusion of macular arterioles and retina arteriovenous communications are visible (black arrow). (B) Retinal arteriolar occlusions have worsened in the temporal periphery. communications, and avascular peripheral retina with fibroglial proliferation. Thölen $e t$ $a l^{4}$ described a young man whose routine eye examination showed narrowing of a superotemporal retinal arteriole with irregular diameter of the corresponding vein, fibroglial proliferation, and arteriovenous communications. These authors suggested the possibility of either a primary occlusion of a retinal arterial or venous branch or alterations of vessel development. In contrast with other reported cases, our patient presented with acute unilateral occlusion of macular arterioles, combined with diffuse retinal arterial occlusive disease, in adulthood. Panretinal photocoagulation may have prevented fibroglial proliferation which was described in previous reported cases. Recent reports suggest that pathogenesis of NF-l vasculopathy may result from dysfunction of neurofibromin, the NF-1 gene product, in vessel endothelial and smooth muscle cells.

Our case shows that NF-1 related occlusive retinal arteriopathy may suddenly appear during adulthood and should be considered in patients with NF-1 or in young adults with retinal vascular occlusive disease and unknown NF-1.

\section{A Lecleire-Collet, S Y Cohen, C Vignal,}

A Gaudric

Department of Ophthalmology, Hôpital Lariboisière Assistance Publique-Hôpitaux de Paris and Université Paris 7, France

A Lecleire-Collet

Department of Ophthalmology Rouen University Hospital Charles Nicolle, Roven, France

S Y Cohen, G Quentel

Centre Ophtalmologique $\mathrm{d}^{\prime}$ Imagerie et de Laser, Paris, France

Correspondence to: Dr Salomon Y Cohen, Centre Ophtalmologique d'Imagerie et de Laser, 11 Rue Antoine Bourdelle, 75015 Paris, France; sycohen@ club-internet.fr

doi: 10.1136/bjo.2005.077701

Accepted for publication 12 August 2005

\section{References}

1 Saylor WR, Saylor DC. The vascular lesions of neurofibromatosis. Angiology 1974;25:510-19.

2 Moadel K, Yannuzzi LA, Ho AC, et al. Retinal vascular occlusive disease in a child with neurofibromatosis. Arch Ophthalmol 1994; 112:1021-3.

3 Kadoi C, Nagaki Y, Hayasaka S. Unilateral peripheral refinal vascular occlusion in a young Japanese woman with neurofibromatosis- 1 . Retina 2003;23:541-3.

4 Thölen AM, Messmer EP, Landau K. Peripheral retinal vascular occlusive disorder in a young patient with neurofibromatosis 1 . Retina 1998:18:184-6.

5 Hamilton SJ, Friedman JM. Insights into the pathogenesis of neurofibromatosis 1 vasculopathy. Clin Genet 2000;58:341-4.

\section{Are biometric parameters a risk} factor for idiopathic macular hole formation? Results of a matched case-control series

Recognised risk factors for the formation of idiopathic full thickness macular holes (IFTMH) include age, female, sex, and high myopia. ${ }^{12}$ However, we noticed that patients with IFTMH within our population, tended to have shorter than normal axial lengths. An age and sex matched case-control study was 
therefore performed to test this hypothesis and determine whether biometric factors may be associated with formation of IFTMH.

\section{Methods}

Consecutive patients undergoing surgery for IFTMH had age, sex, axial length in millimetres $(\mathrm{mm})$, and corneal keratometry $(\mathrm{K})$ measurements in dioptres (D) recorded.

For each case three age and sex matched controls were randomly identified from a cataract surgery database (Medisoft). Biometric measurements were made in all cases and controls using the IOL-Master (Zeiss, Jena). Mean corneal curvature was calculated by averaging the two K readings.

Descriptive statistics and conditional logistic regression method for analysis of matched pair data were used to infer estimates.

\section{Results}

Thirty five consecutive cases and 105 controls were identified with a mean age of 67.3 years (35-77 years) and a female to male ratio of 24:11. The mean (SD) axial length in the cases was $22.94(0.96) \mathrm{mm}$ whereas in controls it was $23.48(1.44) \mathrm{mm}$. The axial lengths of the cases also fell within a narrower range than those of the controls (fig 1). Mean K readings in cases was $44.04 \mathrm{D}$ (range 41.87-46) and 43.44 D in controls. (range 40.56-47.92).

Evidence suggests that the longer the axial length the less the odds of being a case; odds ratio (OR) $0.67(\mathrm{p}=0.036$, CI 0.47 to 0.77 ) and the higher the dioptre power of the curvature the higher the odds of being a case as opposed to a control; OR 1.50 ( $\mathrm{p}=0.02$, CI 1.07 to 2.01 ).

\section{Comment}

As in previous studies our population sample demonstrates an increased risk of IFTMH in females with most cases clustering in the 6070 year age group. ${ }^{3}{ }^{4}$ The mean axial length in controls was also found to be similar to results in the published literature. ${ }^{56}$

Mean spherical equivalence (MSE) was measured by the Eye Disease Case Control Study Group ${ }^{7}$ and although their findings were statistically insignificant, their regression analysis found an increased likelihood of hypermetropia in the IFTMH cases. Our cases clustered in a relatively narrow range of axial lengths $(21 \mathrm{~mm}-25.31 \mathrm{~mm}$ in the cases compared to $20.64 \mathrm{~mm}-29.48 \mathrm{~mm}$ in the controls) with the maximum axial length in the cases being $25.31 \mathrm{~mm}$ (fig 1). In Japanese literature, high myopia is a prominent, recognised risk factor and in one study the overall mean MSE was -0.66 D. $^{2}$ From our study it appears myopic macular holes are encountered much less frequently in a predominantly white population.

We believe the significant corneal dioptric differences found may be a result of the natural process of emmetropisation of a shorter eye, or alternatively be an independent risk factor.

IFTMH are thought to arise from retinal surface traction which in turn arises from an abnormal and incomplete posterior vitreous detachment. $^{8}$ Our results suggest that eyes with shorter than average axial lengths and therefore lower volume vitreous cavity are either at increased risk of developing such incomplete and abnormal vitreous detachments or, in the presence of such an incomplete vitreous detachment, of developing a macular hole. We are not aware that shorter axial length has previously been shown to be significantly associated with IFTMH.

S P Shah

Vitreoretinal Speciality, Eye Department, St Thomas's Hospital, Lambeth Palace Road, London' SEI 7EH, UK

C Bunce Moorfields Eye Hospital, London, UK

R L Johnston

Cheltenham General Hospital, Cheltenham, Sandford Road, Cheltenham GL53 7AN, UK

D A H Laidlaw

St Thomas's Hospital, Lambeth Palace Road, London SEI 7EH, UK

Correspondence to: Shaheen P Shah, MRCOphth, Ophthalmology, Vitreoretinal Speciality, Eye Department, St Thomas's Hospital, Lambeth Palace Road, London SEI 7EH, UK shaheen_shah@hotmail.com

doi: 10.1136/bjo.2005.077008

Accepted for publication 21 August 2005

RU has a commercial and proprietary interest in the cataract database from which control data were derived.

\section{$\square$ Cases $\square$ Controls}

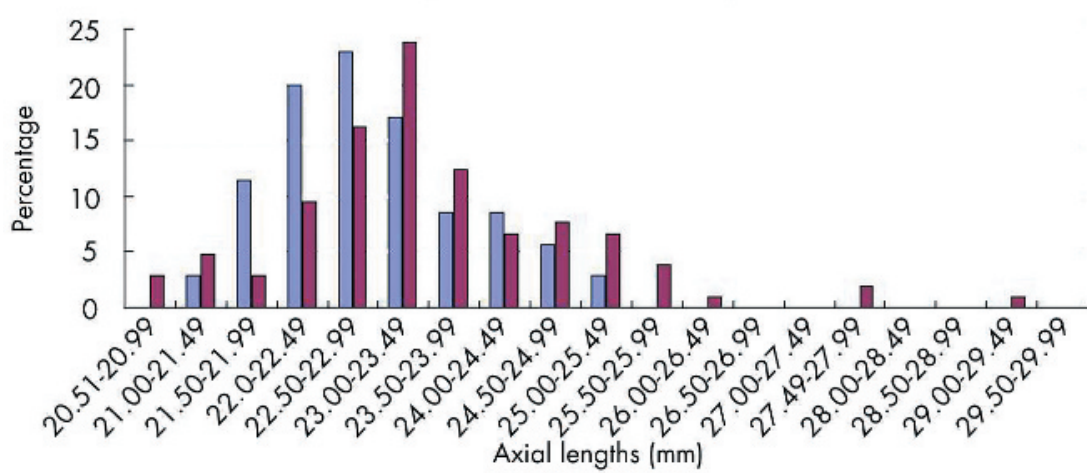

Figure 1 Axial lengths $(\mathrm{mm})$ of patients with macular holes compared with age and sex matched controls.

\section{References}

1 Evans JR, Schwartz SD, McHugh JD, et al. Systemic risk factors for idiopathic macular holes: a case-control study. Eye 1998;12(Pt 2):256-9.

2 Kumagai K, Ogino N, Demizu S, et al. Clinical features of idiopathic macular holes. Nippon Ganka Gakkai Zasshi 2000;104, 118:19-25.

3 James M, Feman SS. Macular holes. Graefes Arch Clin Exp Ophthalmol 1980;215:59-63.

4 McDonnell PJ, Fine SL, Hillis Al. Clinical features of idiopathic macular cysts and holes. Am J Ophthalmol 1982;93:777-86.

5 Shimmyo M, Orloff PN. Corneal thickness and axial length. Am J Ophthalmol 2005; 139:553-4.

6 Gudmundsdottir E, Arnarsson A, Jonasson F. Five-year refractive changes in an adult population: Reykjavik Eye Study. Ophthalmology 2005; 112:672-7.

7 No authors listed. Eye disease case control study group, Risk factors for idiopathic macular holes. Am J Ophthalmol 1994; 118:754-61.

8 Chauhan D, Antcliff R, Rai P, et al. Papillofoveal traction in macular hole formation: the role of optical coherence tomography. Arch Ophthalmol 2000; 118:32-8

\section{Choroiditis and exudative macular detachments in a post transplant leukaemic patient: an unusual presentation of Pneumocystis jiroveci infection}

Pneumocystis jiroveci is a commensal found in the respiratory tract of healthy individuals. It can cause infections of almost any organ in immunocompromised hosts. P jiroveci choroiditis, featuring multifocal choroidal lesions, has been reported in immunocompromised patients despite prophylactic use of inhaled pentamidine. We report a post-transplant leukaemic patient whose posterior segment findings demonstrate an atypical picture of recurrent $P$ jiroveci choroiditis.

\section{Case report}

A 20 year old woman presented with a l week history of bilateral blurred vision, dyspnoea, and dry cough. Visual acuity was reduced to $6 / 36$ bilaterally with posterior subcapsular cataracts. Funduscopy disclosed multifocal, creamy-yellow choroidal lesions at both posterior poles with exudative retinal detachments at the maculae (fig 1). Optic discs and retinal vessels were normal. Fluorescein angiography showed early patchy hypofluorescence (corresponding to the choroidal lesions) and late hyperfluorescence with pooling beneath the detachments.

Three years earlier she had undergone a successful allogeneic bone marrow transplant for chronic myeloid leukaemia. Her maintenance therapy included inhaled pentamidine as prophylaxis against $P$ jiroveci infection. However, she admitted to not taking her inhaled pentamidine. Molecular genetic and radiological investigations excluded leukaemic recurrence. Chest $x$ ray showed left lung shadowing and bronchoalveolar lavage isolated $P$ jiroveci. $P$ jiroveci pneumonia with $P$ jiroveci choroiditis was diagnosed and high dose oral co-trimoxazole $7.2 \mathrm{~g} /$ day $(120 \mathrm{mg} / \mathrm{kg} /$ day) and prednisolone $60 \mathrm{mg} /$ day commenced. Within 2 weeks her respiratory and ocular symptoms improved. Visual acuities returned to $6 / 9$ and the fundal appearances resolved. She was discharged on daily oral co-trimoxazole $960 \mathrm{mg}$, prednisolone $20 \mathrm{mg}$, and inhaled pentamidine. 